- Gingival topography correlates with support from the teeth and underlying bone architecture.

- Assessing periodontal biotype and bioform is a prerequisite for prosthodontic and implant treatment planning.

- A classification of gingival progression of the anterior maxillary sextant is elementary for creating optimal 'pink aesthetics'.

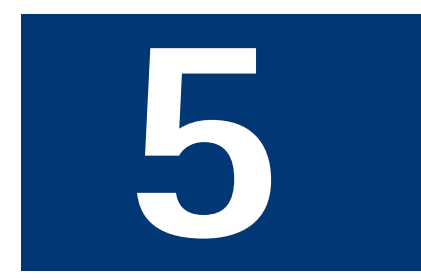

VERIFIABLE CPD PAPER

\title{
Anterior dental aesthetics: Gingival perspective
}

\section{Ahmad ${ }^{1}$}

The purpose of this series is to convey the principles governing our aesthetic senses. Usually meaning visual perception, aesthetics is not merely limited to the ocular apparatus. The concept of aesthetics encompasses both the time-arts such as music, theatre, literature and film, as well as space-arts such as paintings, sculpture and architecture.

\section{ANTERIOR DENTAL AESTHETICS \\ 1. Historical perspective \\ 2. Facial perspective \\ 3. Dento-facial perspective \\ 4. Dental perspective \\ 5. Gingival perspective}

6. Psychological perspective*

* Part 6 available in the $B D J$ book of this series

\section{INTRODUCTION}

The gingival perspective is concerned with the soft tissue envelope surrounding the teeth. The gingival texture, shape, tooth-to-tooth progression and its relation to the extra-oral tissues is interdependent on many factors. These include anatomy of the dentogingival complex, tissue hierarchy, osseous crest considerations, periodontal biotype and bioform, tooth morphology, contact points, tooth position (gingival progression), and extraoral skeletal and soft tissue landmarks. The aim of this section on anterior dental aesthetics is to discuss these variables, citing physiological norms, and clinical protocols to assess and rectify abnormalities for a specific treatment modality.

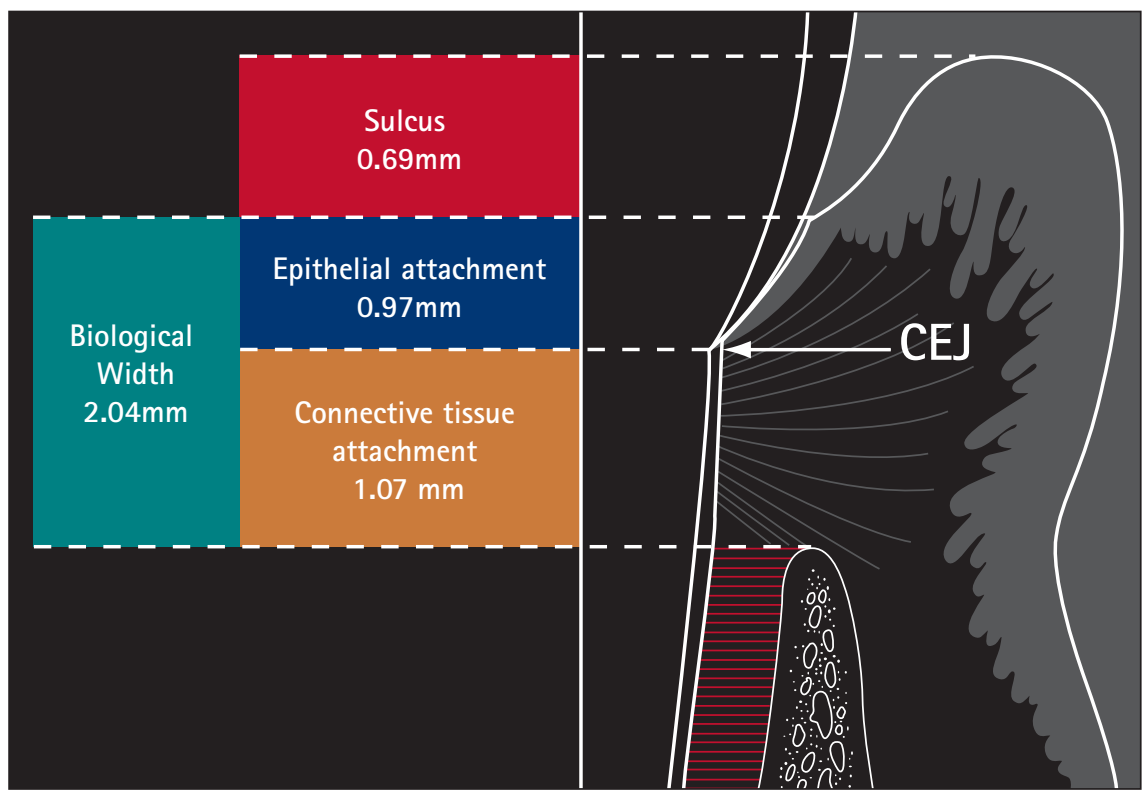

ANATOMY OF THE DENTOGINGIVAL COMPLEX

In cross section, the dentogingival complex is composed of three entities: the supra-crestal connective tissue attachment, epithelial (or junctional epithelium) attachment and the sulcus (Fig. 1). The connective tissue fibres emanate from the osseous crest to the cemento-enamel junction (CEJ), the epithelial attachment from the CEJ onto the tooth enamel, and coronal to the latter is the gingival sulcus or crevice. The landmark research by Garguilo ${ }^{1}$ and Ingber $^{2}$ established the ubiquitously quoted biologic width, calculated by addition of the linear measurement of the connective tissue and epithelial attachment, and quoted as $2.04 \mathrm{~mm}$. The connective tissue and epithelial
BDS, The Ridgeway Dental Surgery, 173 The Ridgeway, North Harrow, Middlesex HA2 7DF, United Kingdom.

Tel: +44 (0)20 88613535 , Fax: +44 (0)20 88616181 www.IrfanAhmadTRDS.co.uk Email:iahmadbds@aol.com

\section{Refereed Paper}

doi: 10.1038/sj.bdj.4812611

$\odot$ British Dental Journal 2005; 199 :

195-202
Fig. 1 Sagittal cross section of the dentogingival complex 
Fig. 2 Facial view of the dentogingival complex showing the mucosa, mucogingival junction attached gingivae and the free gingival margin (FGM) attachments have been shown to be the most consistent, while the sulcus depth varies enormously, depending on type of tooth, site of the tooth, presence or absence of adjacent teeth, diastemae, periodontal disease and passive or altered passive eruption. The significance of the biologic 'width' is its presence around every tooth. It is also worth noting that the biologic 'width' is actually a threedimensional concept, not limited to a single linear plane, and the term 'biologic space' has been proposed to encompass this three-dimensional form. In effect, this natural barrier, or shield protects the two most vulnerable structures of a tooth; the periodontal ligament and alveolar bone, which ultimately determines the survival and longevity of the dental elements. Of course, a shield is most effective when intact, if violated by bacteria or iatrogenic insult, its function is severely compromised, which jeopardises the teeth causing a precarious outcome. For example, infestation by pathogens is a precursor to periodontal disease, while violation by restorative procedures, including surgery or crown preparation stages, mitigates the efficacy of this buffer zone.

From the facial view, a healthy periodontium has the following demarcations. Starting apically, the loose mucosa terminates at the mucogingival junction. From this point coronally, the tissue is keratinised and divided into the attached gingivae, which terminates as the free gingival margin (FGM) (Fig. 2). The texture of the keratinised gingivae can be highly stippled or smooth (Figs 3 and 4), or have intermediate textures between these two extremes. In certain cases, there is a distinct elevation of the FGM called the gingival groove (Fig. 5), while individuals with dark complexion often have melanin pigmentation of the attached gingivae (Fig. 6).

\section{TISSUE HIERARCHY}

To appreciate the following discussion, it is important to consider tissue hierarchy, or command structure, of the dentogingival complex.

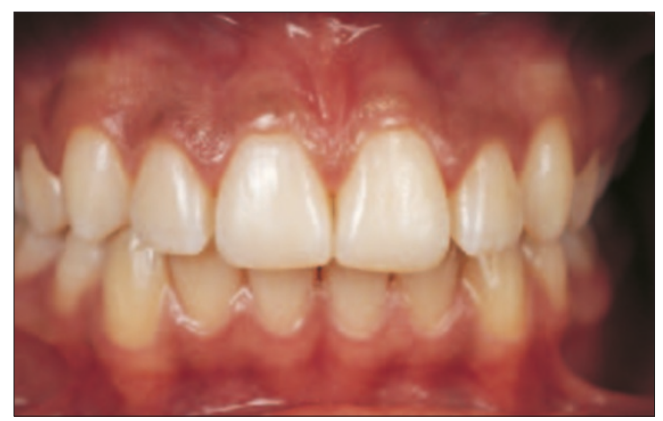

The protagonists are the teeth, which in turn influence the contour of the soft tissue and underlying osseous architecture.

Embryologically, the bone and soft tissues develop first, and lastly the teeth erupt into the oral cavity through the bone/soft tissue envelope. Because of this sequence, it is fallacious to assume that the hierarchy is concurrent with the chronological development of the three tissues comprising the dentogingival unit. For example, consider a diastema

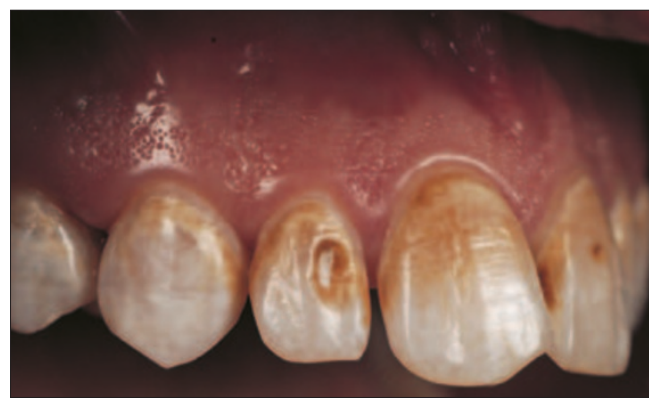

Fig. 3 Highly stippled texture of the attached gingivae



Fig. 4 Smooth texture of the attached gingivae

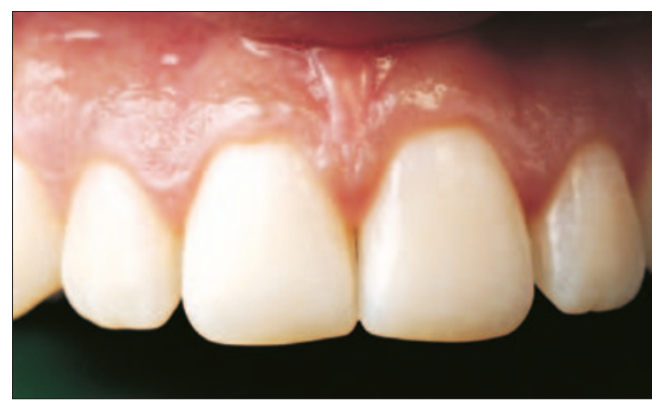

Fig. 5 Elevation of the FGM resulting in the so-called gingival groove

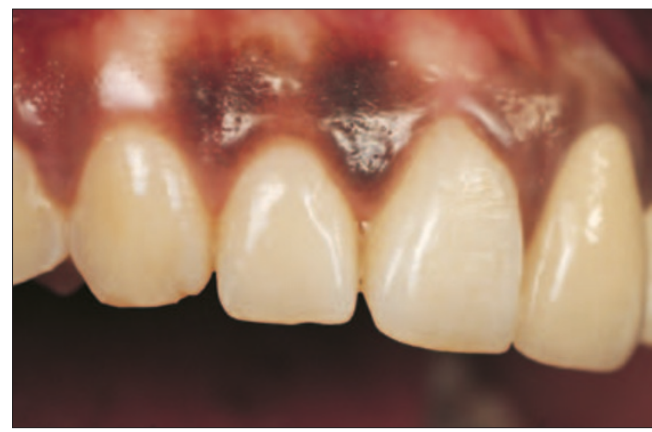

Fig. 6 Physiological pigmentation of the attached gingivae of a dark skinned individual

between natural teeth. If the hierarchy was reversed, the interproximal osseous crest would peak $3 \mathrm{~mm}$ from the incisal edge (assuming a normal osseous bone pattern), and the soft tissue would cover the latter, creating an interdental papilla, terminating at the incisal edges between the teeth. Clearly, this is not the case. It is the diastema between the teeth that determines the soft tissue level, which terminates apically and the underlying bone positions itself accordingly. Another example is the distance from the contact point to the interproximal osseous crest. If the dis- 
crepancy between the contact point and osseous peak is greater than $5 \mathrm{~mm}$, there is incomplete papilla fill. However, if this distance is $5 \mathrm{~mm}$ or less, a papilla completely fills the interproximal void, but the interproximal bone does not take a position $3 \mathrm{~mm}$ from the most coronal aspect of the papilla. This again emphasises the dictum that the teeth are the protagonists, followed by the soft tissue and bone topography (Figs 7 and 8).

\section{OSSEOUS CREST}

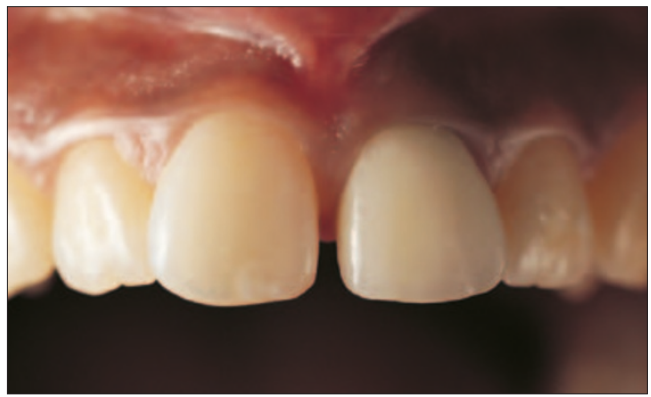

Fig. 7 Maxillary median diastema lacking an interproximal papilla

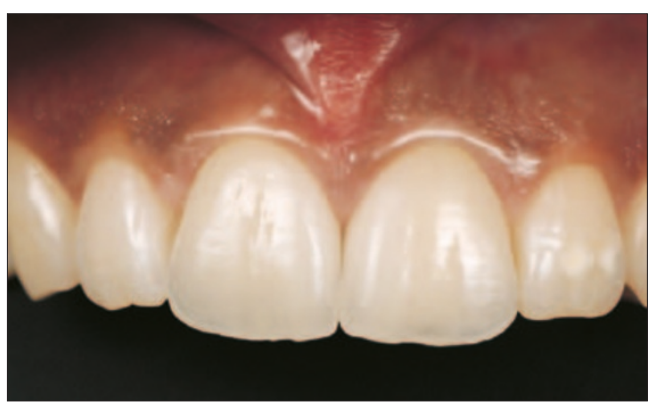

Fig. 8 Same patient as Fig. 8 following the provision of two all-ceramic crowns for the maxillary central incisors. The contact point created between the crowns is now less than $5 \mathrm{~mm}$ from the interproximal osseous crest. allowing the formation of an interdental papilla

The quoted measurement of $2.04 \mathrm{~mm}$ for the biologic width assumes that the osseous crest is normal, prevalent in $85 \%$ of the population, with a sulcus depth of $1 \mathrm{~mm}$, yielding $3 \mathrm{~mm}$ for the entire dentogingival complex. The importance of the latter is that if crowns are necessary, meticulous care should be taken to ensure that this equilibrium is maintained ( $3 \mathrm{~mm})$, and not violated by any clinical procedure (Figs 912). For implant placement, a different protocol is adopted. If the preoperative dentogingival complex is normal ( $3 \mathrm{~mm}$ ), and the tooth requires extraction and subsequent implant replacement, it becomes necessary to employ orthodontic extrusion to compensate for post surgical recession (2-3 mm). In 13\% of individuals, the osseous crest is low, and the sulcus measurement exceeds the $1 \mathrm{~mm}$ norm, varying from 2-4 mm mid-facially and greater than 4 mm interproximally. In these cases, the deep gingival crevice is particularly delicate and susceptible to surgical manipulation and ensuing recession. On the other hand, in $2 \%$ of patients, the bone crest is high, with a sulcus depth of less

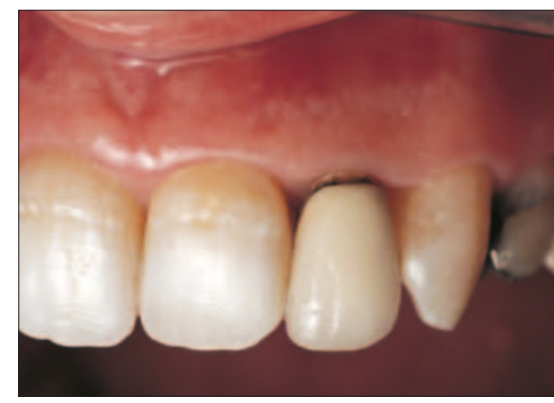

Fig. 9 Preoperative defective porcelain fused to metal crown on maxillary left lateral incisor

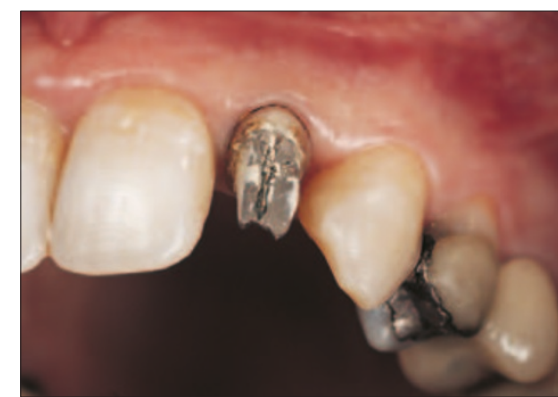

Fig. 11 Intra-crevicular placement of retraction cord to protect the biological width during crown preparation

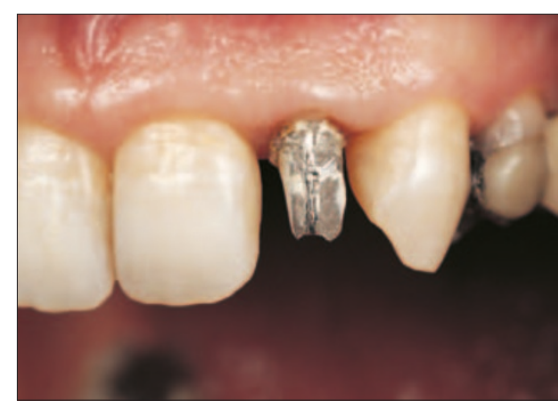

Fig. 10 Removal of offending crown, revealing an intra-radicular cast post and core

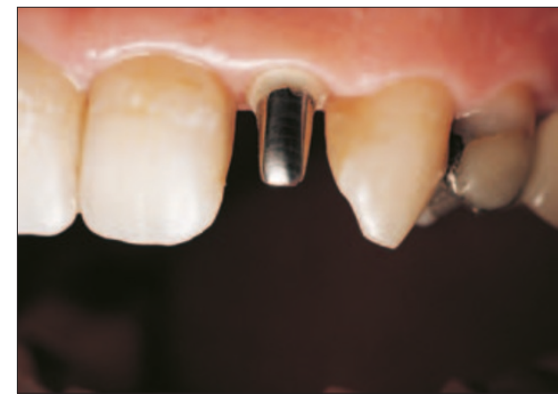

Fig. 12 Adopting a haemorrhage free clinical protocol to refine and shape the metal core and tooth margins

than $1 \mathrm{~mm}$, creating a perilous threat to biological width violation by restorative procedures such as crown preparation and subgingival crown margin location.

\section{PERIODONTAL BIOTYPE AND BIOFORM}

The human tissue biotype is classified as thin, normal or thick (Figs 13 and 14). ${ }^{3,4}$ The thin periodontal biotypes are friable, escalating the risk of recession following crown preparation and periodontal or implant surgery. This is particularly significant for full coverage crowns for the following reasons. Firstly, the thin gingival margins allow visibility of a metal substructure (either porcelain fused to a metal crown or implant abutment), thereby compromising aes-

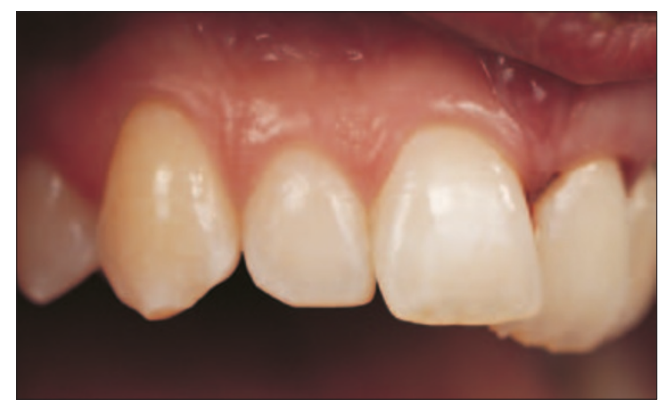

Fig. 13 Thin periodontal biotype

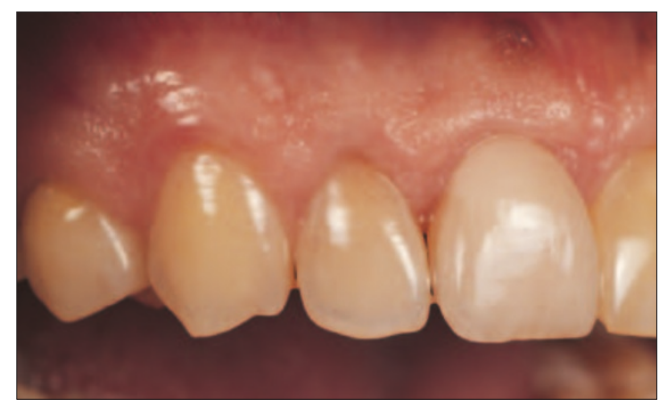


Fig. 15 High scallop periodontal bioform

Fig. 16 Normal scallop periodontal bioform (the maxillary central incisors have acrylic temporary crowns) thetics in the anterior regions of the mouth. In these circumstances, all-ceramic crowns, or ceramic implant abutments are a prerequisite to avoid aesthetic reproval. Secondly, due to the fragility of the thin tissue, delicate management is essential for avoiding recession and hence visibility of subgingivally placed crown margins at the restoration/tooth interface. Conversely, a thick biotype is fibrotic and resilient, making it resistant to surgical procedures with a tendency for pocket formation (as opposed to recession). Therefore, a thick biotype is more conducive for implant placement, resulting in favourable aesthetic outcomes.

Periodontal bioforms are categorised into three basic gingival scallop morphologies, high, normal and flat (Figs 15-17). While the facial and lingual gingival scallop mimics the underlying bone architecture, this is not always the case interproximally. The norm is a discrepancy of $4 \mathrm{~mm}$ between the interproximal gingival peaks (most coronal) and the mid-facial free gingival margin peaks (most apical). When this discrepancy is less than $4 \mathrm{~mm}$, a flat scallop is evident, while a greater than $4 \mathrm{~mm}$ discrepancy results in an exaggerated or high scallop. With a shallow scallop, the interproximal bone is thin, and the interproximal gingival contour nearly parallel to the underlying bone contour. The latter is advantageous for implant therapy since the bone has a congruous relationship with the FGM and is less prone to post-surgical recession. With a pronounced or lhigh scallop, the interproximal
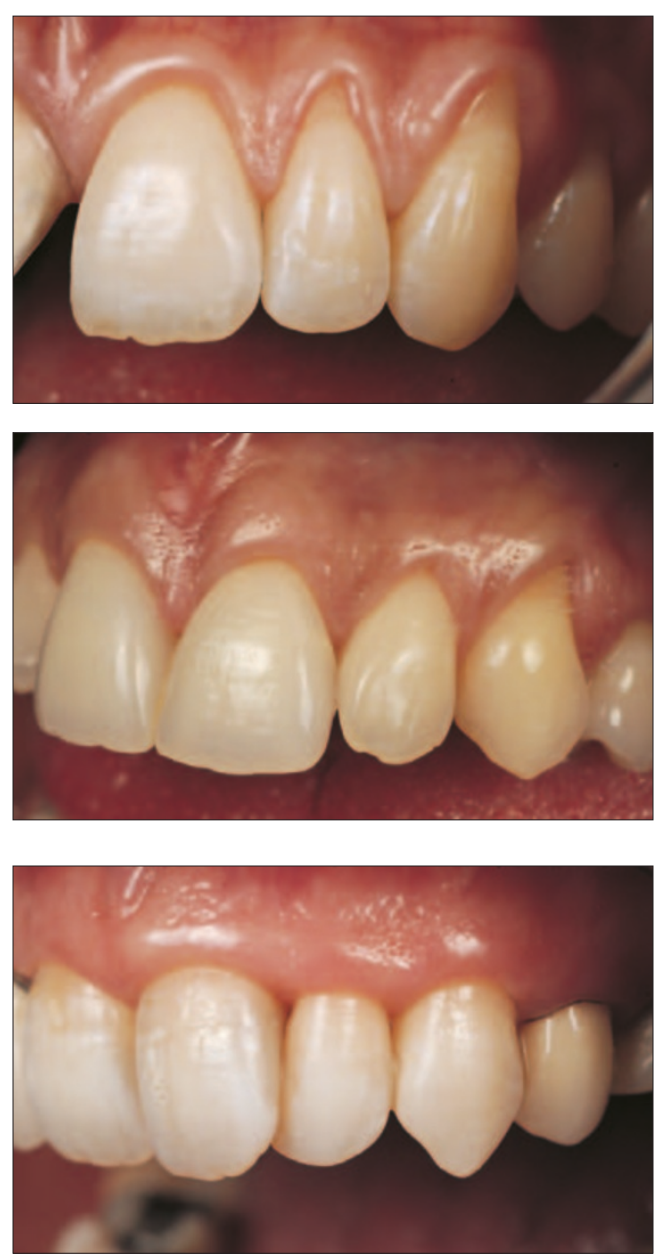

bone is wider, but the disparity between the bone contour and the FGM is problematic for favourable aesthetics (due to possible recession and creation of 'lack triangles') following implant or restorative procedures. ${ }^{5}$

\section{TOOTH MORPHOLOGY}

Tooth morphology determines two aspects of gingival undulations. Firstly, the basic tooth forms: circular, square or triangular, determine the degree of gingival scallop. Circular (oval) or square teeth produce a shallower gingival scallop, while triangular teeth form the opposite, a pronounced scallop. The latter predisposes to the so-called 'black triangles'; especially with a thin biotype which has a propensity for recession. Furthermore, triangular teeth have divergent roots with thicker interproximal bone, resulting in reduced vertical bone loss compared with square teeth, whose root proximity and thinner interdental bone have a higher incidence of vertical bone resorption. However, squarer teeth yield better interproximal papilla maintenance due to a smaller interproximal distance from the osseous crest to the FGM.

Secondly, the convex acuity of a tooth circum-

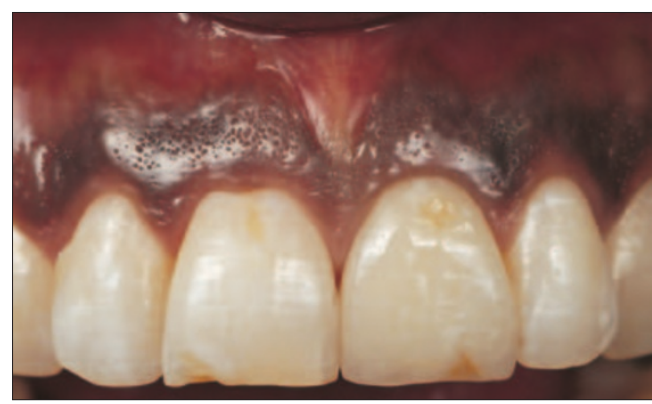

Fig. 18 The convex acuity of tooth topography determines the position of the FGM. The gingival zenith on the left central incisor is apical and distal to the long axis of the tooth (normal). However, the concave facial profile of the right central incisor has resulted in a coronal mid-facial creep of the FGM

ference influences the coronal/apical position of the FGM. Put succinctly, convex tooth morphology yields a more apical location of the FGM, while a concave shape leads to a coronal position of the FGM (Fig. 18). This is blatantly evident in the gingival zeniths of the maxillary anterior teeth. For a central incisor, the most convex part facially is distal to the long axis of the tooth, with the FGM zenith occupying a similar location. For a lateral incisor, the maximum convexity is in line with the long axis of the tooth, with the gingival zenith positioned accordingly. The canine has a similar maximum convexity to the central incisor, and its gingival zenith is distal to the long axis of the tooth (Fig. 19). Often, poor dentistry conceals these zeniths (Fig. 20), which are readily regained by replacing the offending restoration. The biological relationship of tooth morphology to FGM locations can be exploited to manipulate the soft tissue around prostheses that are aesthetically sensitive. A flat or slightly concave topography of an artificial prosthesis encourages a more corona location of the FGM, while the opposite is true for 


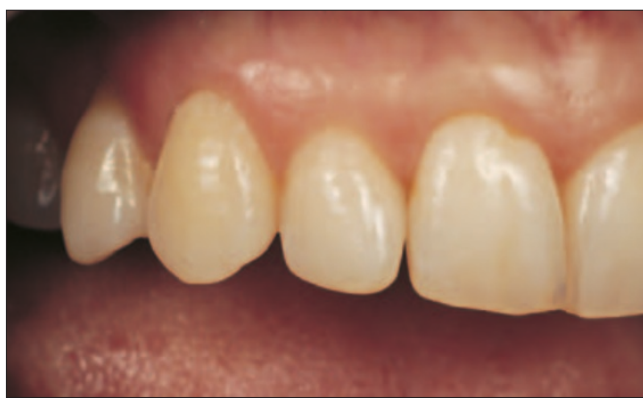

Fig. 19 The gingival zeniths of the maxillary anterior teeth. For the central incisor, the apical peak of the FGM is distal to the long axis of tooth, for the lateral in line with long axis of the tooth, and for the canine distal to long axis of the tooth

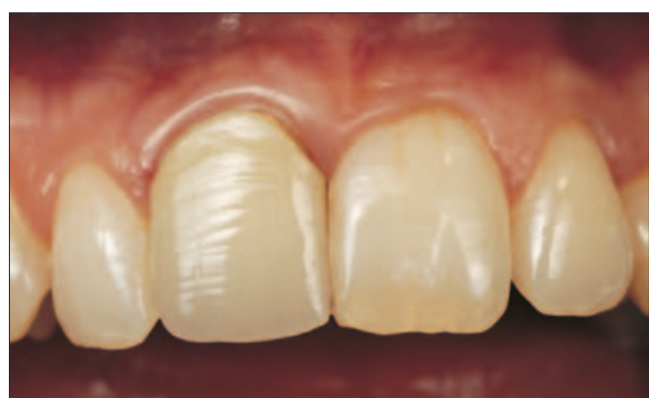

Fig. 20 Defective crown on right central incisor creating an amorphous gingival margin, compared to the left natural central whose gingival zenith is distal to the long axis of the tooth

a convex surface topography, resulting in a more apical position of the FGM. ${ }^{6}$

\section{CONTACT POINTS}

The contact points of the maxillary teeth are relevant for ensuring optimal 'pink aesthetics' for patients with a high smile line (or visible cervical margins). The iconic study by Tarnow, ${ }^{7}$ which produced the ' $5 \mathrm{~mm}$ rule', states that when the distance from the contact point to the interproximal osseous crest is $5 \mathrm{~mm}$ or less, there is complete fill of the gingival embrasures with an interdental papilla. For every $1 \mathrm{~mm}$ above $5 \mathrm{~mm}$, the chance of complete fill is progressively reduced by $50 \%$. For square-shaped teeth with wide contact points, the chances of "black triangles' is minimal compared with triangular teeth having narrow, more incisally positioned contact points (Fig. 21). The existence of the interdental papilla is dependent on the presence or absence

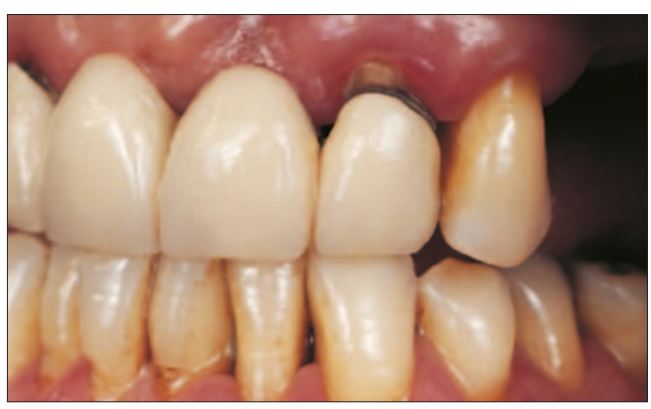

Fig. 21 Defective crown on maxillary left lateral incisor with open gingival embrasures forming the so-called 'black triangles' of teeth. No teeth, no papillae; a scenario, which is evident in edentulous ridges where papillae are absent. For example, if a lateral incisor is extracted, the interproximal papilla between the central incisor and canine will disappear, and the FGM of the lateral incisor will re-establish itself to $3 \mathrm{~mm}$ from the underlying osseous crest. This is identical to the unsupported dentogingival complex on the mid-facial aspect of natural teeth. ${ }^{8}$ The latter is also true for diastemae, where an interdental papilla is absent (Figs 7 and 8). Numerous techniques for preserving ${ }^{9}$ and restoring ${ }^{10}$ the interdental papilla have been described and the prosthodontist should strive to fill open gingival embrasures to create optimal 'pink aesthetics'.

Furthermore, the degree of interproximal fill is also dependant on the periodontal biotype. A thick periodontal biotype encourages interdental fill, while a thinner tissue type creates un-aesthetic hollow gingival embrasures. This problem is compounded when an implant is placed next to a natural tooth. It is the interproximal bone of the adjacent natural tooth that determines the presence, or absence of a papilla, not the bone surrounding the implant fixture. For thick biotypes, the papilla may be established to normal dimensions of $5 \mathrm{~mm}$, but for thin biotypes, it is difficult to recreate a papilla longer than $4 \mathrm{~mm}$ from the osseous crest. ${ }^{11}$ Finally, the $5 \mathrm{~mm}$ rule is only applicable for adjacent natural teeth or implants bounded by natural dentition. Due to the flat fixture platforms, adjacent implants lack the interproximal osseous peak present between natural teeth. ${ }^{12}$ The recently introduced scalloped platform fixtures (Nobel Perfect, Nobel Biocare, Sweden), may help to redress this issue with an endeavour to mimic natural root morphology.

\section{TOOTH POSITION AND GINGIVAL \\ PROGRESSION}

The intra-arch tooth position is assessed in three planes: vertical (apical-coronal), sagittal (faciallingual) and horizontal (mesial-distal). In the vertical plane, the cervical portion of the tooth can be apical, coronal or in line with the FGM. An example of a coronally positioned FGM occurs in cases of altered passive eruption, where the FGM fails to migrate apically to assume its relative position to the CEJ (Fig. 22). The latter position is misleading when placing crown margins subgingivally. If this gingival anomaly is ignored, following crown cementa-

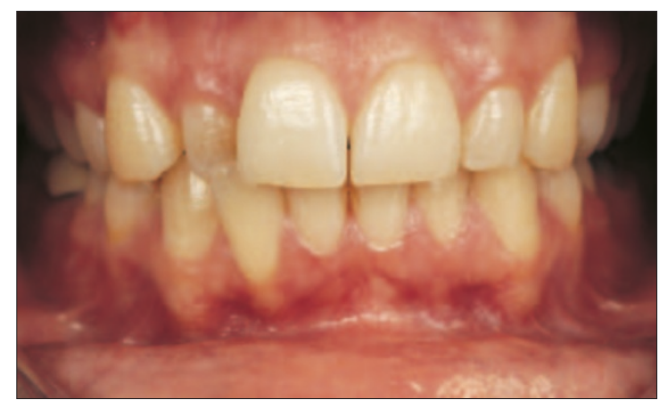

Fig. 22 Altered passive eruption on the maxillary right lateral incisor 
Fig. 23 Bucally placed maxillary left lateral incisor with an apical FGM and thin underlying buccal osseous plate (compare to right lateral incisor)

Fig. 24 Severe crowding of the maxillary anterior sextant tion, the FGM may recede apically, exposing the crown/tooth interface and compromising aesthetics. In the sagittal plane, a facially placed tooth will have an apically located FGM with a thin underlying buccal plate (Fig. 23). In these circumstances, the thin bone contradicts orthodontic repositioning and bone and/or soft tissue grafting may be the only option for rectifying soft tissue contours. Conversely, a lingually placed tooth will reveal a coronally placed FGM, similar to that of altered passive eruption. Finally, imbrications in the horizontal plane result in crowding, due to limited arch space for wide teeth (Fig. 24). These teeth have close root proximity with thin interproximal bone. Orthodontic movement is an option for paralleling roots and creating a more favourable interproximal bone support for the overlying gingival architecture. The opposite is the case for diastemae with thick interproximal bone and blunted papillae. The latter clinical manifestations should be borne in mind when restorative or surgical procedures are undertaken, with a view to predicting the ensuing post treatment gingival levels.

One of the most significant features of gingi-
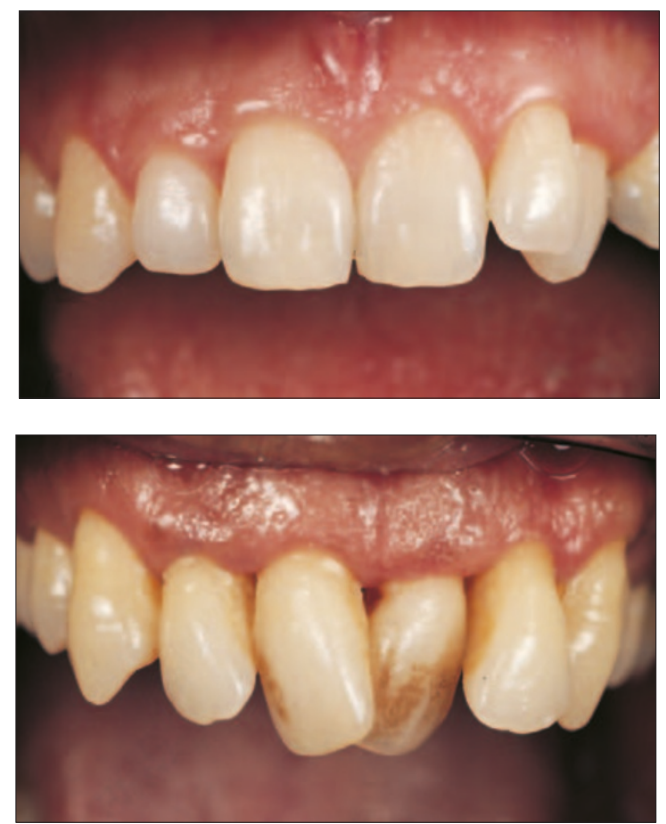

val aesthetics is contour progression from the incisors to canine. The gingival aesthetic line (GAL) is a classification for creating pleasing gingival level transition between the maxillary anterior teeth. GAL is defined as a line joining the tangents of the zeniths of the FGMs of the central incisor and canine. The GAL angle is that formed at the intersection of this line to the maxillary dental midline (Fig. 25). Assuming a normal w/l ratio, anatomy, position and alignment of the anterior dental segment, four classes of GAL are described:

Class I: The GAL angle is between $45^{\circ}$ and $90^{\circ}$ and the lateral incisor is touching or below (1$2 \mathrm{~mm}$ ) the GAL - (Fig. 26)

Class II: The GAL angle is between $45^{\circ}$ and $90^{\circ}$ but the lateral incisor is above (1-2 $\mathrm{mm})$ the GAL and its mesial part overlaps the distal

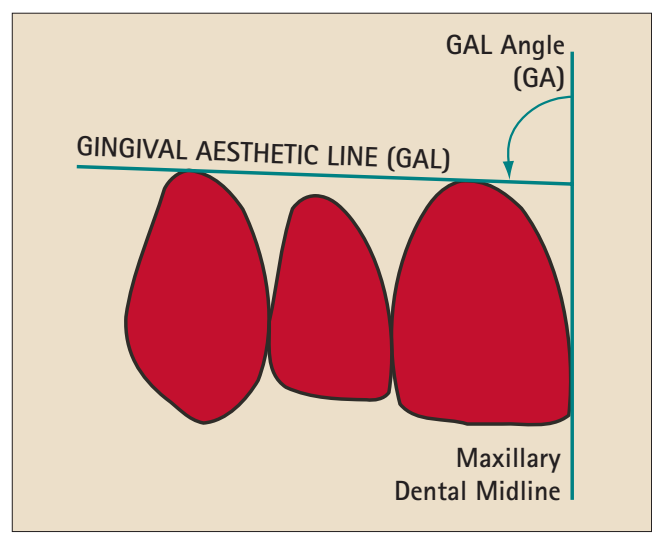

Fig. 25 GAL classification

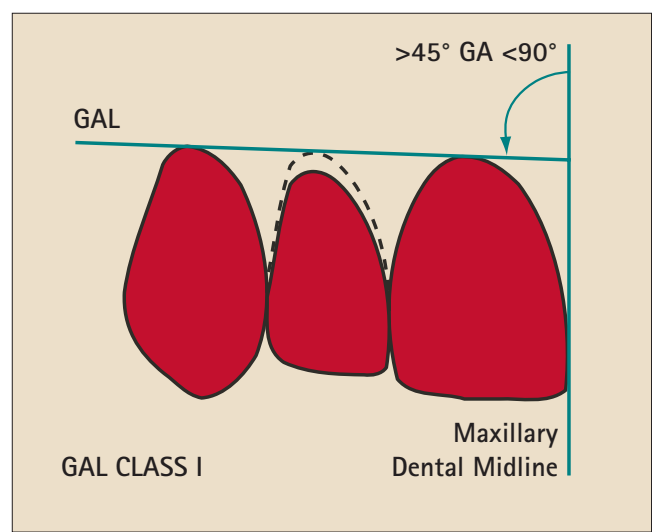

Fig. 26 GAL Class I

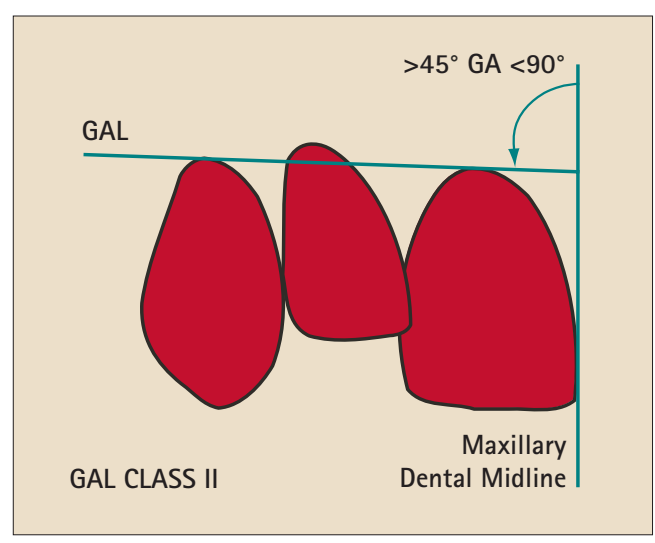

Fig. 27 GAL Class II

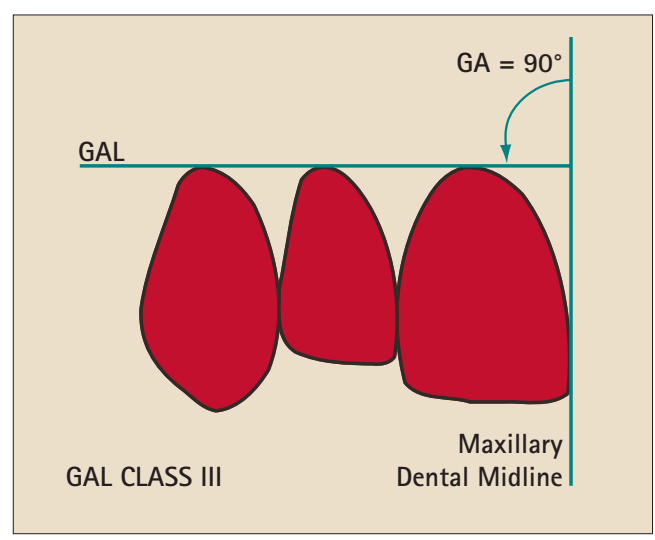

Fig. 28 GAL Class III 
aspect of the central incisor. This situation is often seen in Angle's Class II or pseudo-Class II conditions, and adds variety to the dental composition - (Fig. 27)

Class III: The GAL angle $=90^{\circ}$, and the canine, lateral and central incisors all lie below the GAL - (Fig. 28)

Class IV: The gingival contour cannot be assigned to any of the above three classes (Fig. 29). The GAL angle can be acute or obtuse. A myriad gingival asymmetries are apparent clinically including: recession, passive and altered passive eruption, eccentric eruption patterns, loss of interdental papillae, clefts and high frenal insertions.

In a single mouth, the right and left sides can

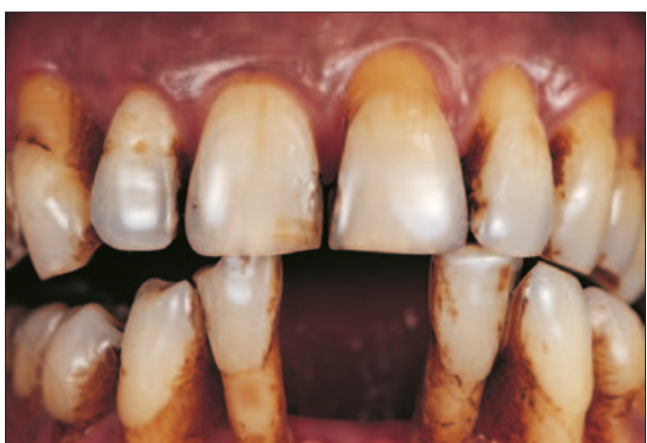

Fig. 29 Erratic gingival progression from central incisor to canine, resulting in a GAL Class IV

display different GAL classes (Figs 30-32). The aim of the clinician is to restore the gingival contour to a GAL Class I, II or III to achieve aesthetic appraisal.

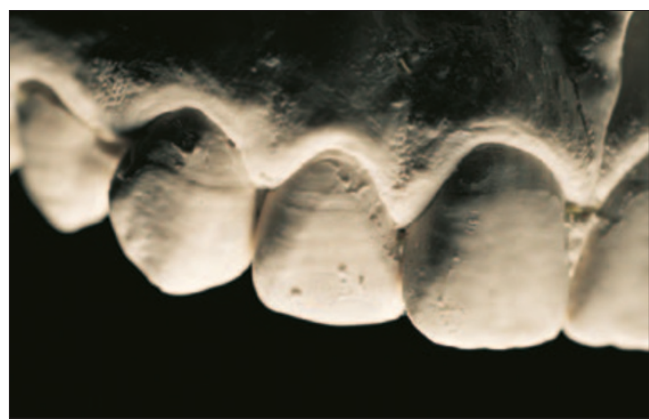

Fig. 30 Right lateral view of maxillary anterior teeth showing a GAL Class III

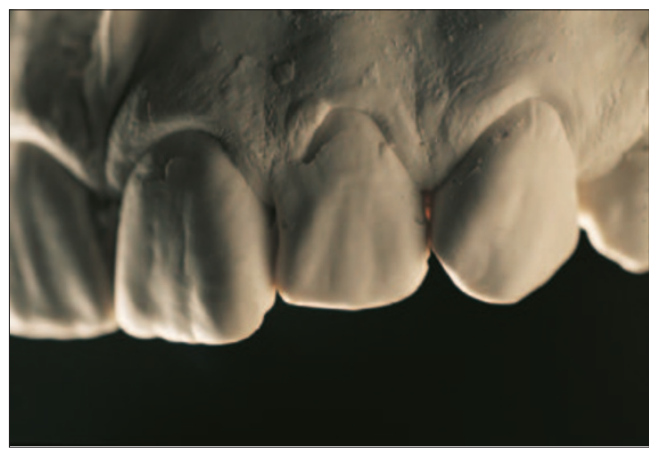

Fig. 31 Left lateral view of maxillary anterior teeth for same patient in Fig. 30, with a GAL Class II

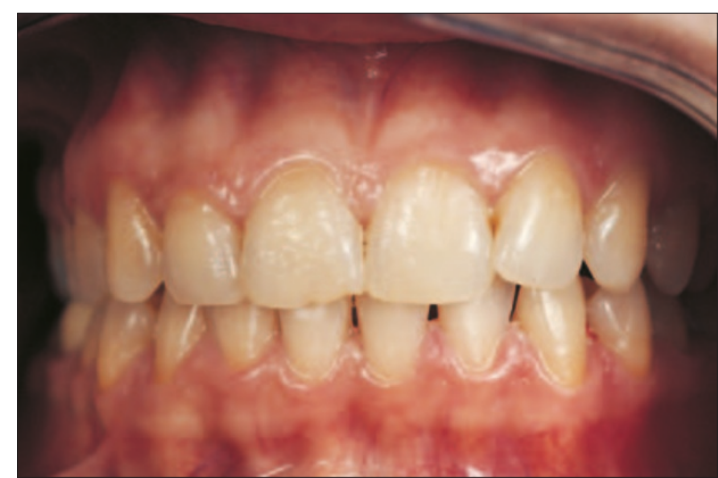

EXTRA-ORAL ANATOMY

Extra-oral anatomy is genetically determined, consisting of the skeletal and soft tissue landmarks. The perio-oral skeletal and soft tissue form of the lower third of the face should harmonise with the dentition. The latter is elaborated in part 2 (facial perspectives) and part 3 (dentofacial perspectives) of this series.

During a relaxed 'ideal smile', the upper lip exposes the cervical aspects of the maxillary anterior teeth. The gingival margins of the maxillary central incisors should be symmetrical and at the same height. Up to $3 \mathrm{~mm}$ of gingival exposure above the cervical margins of the maxillary teeth is aesthetically acceptable (Fig. 33). ${ }^{13}$ Beyond $3 \mathrm{~mm}$ results in a 'gummy' smile, requiring correction by orthodontic or surgical intervention to avoid visual tension (Fig. 34). Treatment modalities depend on the type of pathosis, eg hyperplastic gingivae require gingivectomy or crown lengthening; recession can be corrected using orthodontics or cosmetic periodontal plastic surgery using tissue grafts or guided tissue regeneration membranes; over eruption by orthodontic intrusion; deficient pontic sites by ridge augmentation procedures and skeletal abnormalities by orthognathic surgery.
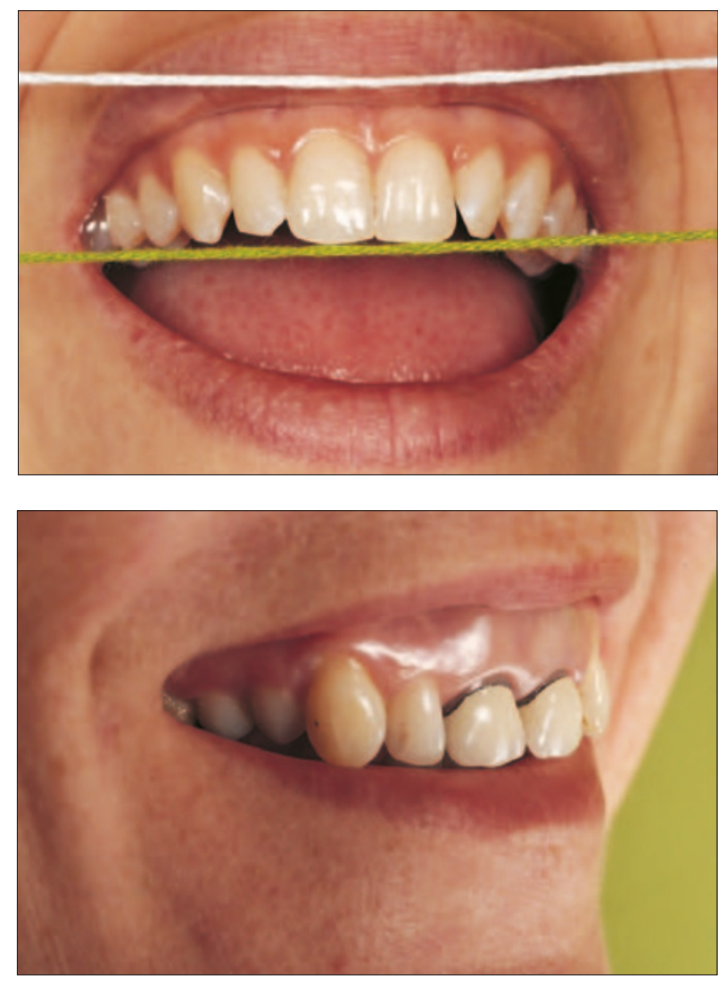

Fig. 32 The right maxillary quadrant shows a GAL Class I, while the left quadrant a GAL Class II

Fig. $33 \mathrm{Up}$ to $3 \mathrm{~mm}$, gingival exposure (white line) during a smile is not detrimental for aesthetic approval. The incisal plane (lime green line) should coincide with the white gingival exposure line

Fig. 34 Excessive gingival exposure results in an unaesthetic 'gummy smile' 


\section{CONCLUSION}

Unlike other topics covered in this anterior aesthetic series (facial, dentofacial or dental), the gingival perspective is the most quantifiable, and least prone to artistic interpretation. However, failing to take account of the soft tissue considerations will negate all the other aspects of treatment, resulting in ultimate aesthetic failure. Correcting gingival anomalies after completing aesthetic rejuvenation is costly, embarrassing and frustrating for the entire dental team. Rather than an afterthought, the gingival viewpoint, at the outset, should be an integral part of any aesthetic treatment planning, ensuring health, approval and longevity of the final result.

1. Garguilo A W, Wentz F M, Orban B. Dimensions and relations of the dentogingival junction in humans. J Periodontol 1961; 32: 321 .

2. Ingber J S, Rose LF, Caslet J G. The biological width concept in periodontics and restorative dentistry. Alpho Omegan 1977; 70: 62-65.

3. Seibet J, Lindhe J. Esthetics and periodontal therapy. In Lindh J, ed. Textbook of clinical periodontology. pp 431-467. Copenhagen: Munksgaard, 1989.
4. Sanavi F, Weisgold AS, Rose LF. Biologic width and its relation to periodontal biotypes. J Esthet Dent 1998; 10 157-163.

5. Kois J C. Altering gingival levels: The restorative connection - Part 1: Biological variables. J Esthet Dent 1994; 6: 3-9.

6. Smukler H, Castellucci F, Capri D. The role of implant housing in obtaining aesthetics: Part 2. Customising the peri-implant soft tissues. Pract Proced Aesthet Dent 2003; 15: 487-490.

7. Tarnow D, Magner A, Fletcher P. The effect of the distance from the contact point to the crest of bone in the presence or absence of the interproximal dental papilla. J Periodontol 1992; 63: 995-996.

8. Spear F. Maintenance of the interdental papilla following anterior tooth removal. Pract Periodont Aesthet Dent 1999; $11: 21-8$

9. Beagle J R. Surgical reconstruction of the interdental papilla: case report. Int J Perio \& Rest Dent 1992; 12: 145-151.

10. Lie T. Periodontal surgery for the maxillary anterior area. Int $J$ Perio \& Rest Dent 1992; 12: 73-82.

11. Jemt T. Regeneration of gingival papilla after singleimplant treatment. Int J Periodont Rest Dent 1997; 17: 327-333.

12. Tranow D, Chu S C, Wallace S S. The effect of inter-implant distance on the height of inter-implant bone crest. J Periodontol 2000; 71: 546-549.

13. Allen P. Use of mucogingival surgical procedures to enhance aesthetics. Dent Clin North Am 1988; 32: 307. 